\title{
A microseismic monitoring pilot project of natural caves in Carajás - PA
}

\author{
*Leonardo Santana de Oliveira Dias (COFFEY), Gerrit Olivier (IMS), Ramon Nunes Araújo (VALE), Marcelo Roberto Barbosa \\ (VALE), luri Brandi (VALE), Mauri Lopes Ferreira (MLF), Marco Antônio da Silva Braga (UFRJ), Romero César Gomes \\ (UFOP)
}

Copyright 2016, SBGf - Sociedade Brasileira de Geofísica

Este texto foi preparado para a apresentação no VII Simpósio Brasileiro de Geofísica, Ouro Preto, 25 a 27 de outubro de 2016. Seu conteúdo foi revisado pelo Comitê Técnico do VII SimBGf mas não necessatamente representa a opinião da SBGf ou Técnico do VII smbGr, mas nao necessariañonte representa a opináo da s de seus associados. E probida a reprodução total ou parcial deste material para propósitos comerciais sem prévia autorização da SBGf.

\section{Abstract}

The mine operations in Carajás - PA face the challenge of producing iron ore without causing any physical damages to the natural caves surrounding the mining areas. To support the sustainable relationship between ore production and environmental constraints, a pilot project to test microseismic monitoring techniques was performed close to a single cave within the area of operation. The acquisition and treatment of 48 hours of continuous seismic data, before and after the execution of two blasts $500 \mathrm{~m}$ away, has showed that the use of this type of technology is well suited to monitor shallow natural caves located inside mining operational areas, indicating unfavorable geological structures present in the caves and enabling to monitor that structures behaviors over time.

\section{Introduction}

At Carajás mine in Brazil, many caves exist between iron rich deposits and other banded iron formation. Although the locations of the caves are well known, due to cultural and historical reasons mining can not disturb or damage these caves.

The challenge facing mining operations is to get closer to environmental constrains, like natural caves, without cause any type of physical damages. Microseismic monitoring technology is ideal to monitor areas where induced seismicity is the source of the changes in natural stress-strain conditions in the earth.

Microseismic monitoring is the measurement of small earthquakes, mostly induced by human activity (like mining) in an industrial setting. When these small earthquakes or microseismic events occurs, the failure of the rock mass releases energy in the form of seismic waves. These seismic waves cause shaking of the surrounding environment, but many of these microseismic events cause shaking so weak that it can not be felt by humans and can only be detected by very sensitive seismic sensors - geophones or accelerometers.

A major advantage of microseismic monitoring over other geotechnical techniques (like crack displacement meters) is that microseismic events can be detected from fractures quite far away whereas crack displacement meters only measure local displacements. If a failure occurs in a location in a cave where there are no crack displacement meters, we would not be able to detect it with these instruments. However, microseismic sensors form an array that is sensitive to fracture within their volume and would be able to detect failures over larger distances. To monitor a single cave, an array of 4 to 8 seismic sensors would be required. To give the same type of coverage, many more crack displacement meters would be necessary.

To examine if state-of-the-art seismic monitoring technology can be used to monitor the response of a specific cave in Carajás, a pilot project was performed with the goal to monitor the microseismic responses of the N4E_0026 cave to mining activities and blasts.

For this pilot project 5 sensors were installed in an accessible area close to N4E_0026 and recorded continuous data for 48 hours. During this time, two blasts were performed roughly $500 \mathrm{~m}$ away.

This article presents the background details on the techniques used to analyze the effects of the mining activities on the cave, besides show that in this case, the blasts had no permanent effect on the cave and only induced temporary weakening in an area $100 \mathrm{~m}$ to the north of the cave.

\section{Methodology}

To investigate the potential results of microseismic monitoring in mining areas with cave protection restrictions, a monitoring campaign of one week was executed and a 4 temporary surface seismic sensors and 1 permanent borehole sensor was installed and data was recorded continuously for 48 hours. During this time, two blasting sequences were performed roughly $500 \mathrm{~m}$ away (Figure 1 and Figure 2).

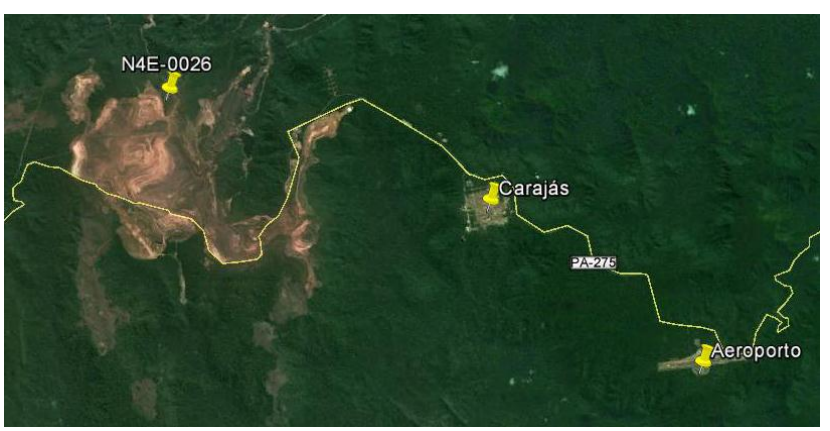

Figure 1 - N4E_0026 location (Google Earth. Version 7.1.5.1557, access on April $4^{\text {th }}$ ). 


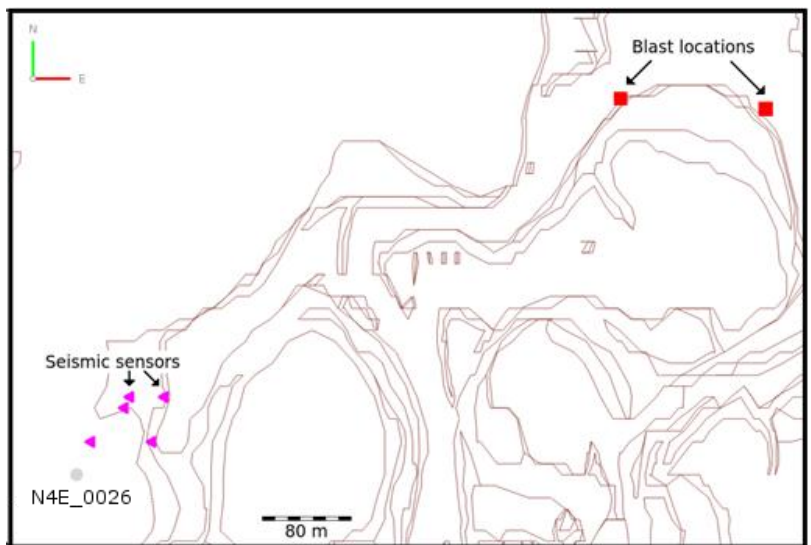

Figure 2- The location of the seismic sensors and cave relative to the mining areas and blast. The sensors were located between 40 and $100 \mathrm{~m}$ away from the cave and the blasts were conducted more than $500 \mathrm{~m}$ away.

Two different microseismic methods were performed over the data acquisition campaign: 1) conventional microseismic monitoring and 2) continuous passive monitoring.

Conventional microseismic evaluates the microseismic activities of an area through the determination of when the rock mass damage occurs, where they are located and what their extension (degree of impairment).

The fracturing of the rock can be caused by tensile, compressive or shear forces. The location and energy released by the fracture can be determined with reasonable accuracy by analyzing the arrival times and characteristics of the vibrations [1].

Continuous passive monitoring is a new technique that has emerged and that can enable to monitor how an area is responding to mining activities even when no failures (or microseismic events) are recorded. This technique is called ambient noise analysis and is particularly useful in an environment where it is not expected that much microseismic activity will occur, such as these shallow zones around the caves.

This method relies on the relationship between the speed at which the seismic waves travel through the medium and how damaged the medium is. The more damaged the medium becomes, the slower the seismic velocity will be.

A simple illustration of this relationship is shown in Figure 3 , where it is possible to see that a blast causes the opening of microfractures that leads to a small decrease in the speed that the seismic waves travel between the two underground sensors.

Following it will be presented the results of the analysis of the acquisition of $100 \mathrm{~GB}$ of data collection.

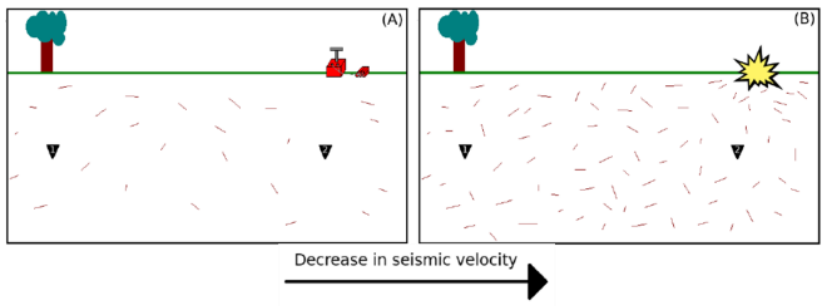

Figure 3 - An illustration showing the dependence of seismic velocity on damage. Here a blast opens fractures and decreases the bulk seismic velocity.

\section{Results}

Two days of continuous seismic data were recorded by 5 geophones installed close to the N4E_0026 cave (see Figure 2). These geophones continuously record the ground motions (or shaking) in the area very accurately and detected ground motions as small as $0.0001 \mathrm{~mm} / \mathrm{s}$. The ground motions were sampled 6000 times per second and saved as seismograms, roughly every 20 seconds.

The geophones were connected to a mobile seismic station. This station digitizes the recorded waveforms and timestamps each ground motion sample and finally records the ground motions measured by the geophones to a USB flash drive (Figure 4). The total amount of data recorded was roughly $100 \mathrm{~GB}$ for the two day period. The mobile seismic station was powered by a $12 \mathrm{~V}$ battery.

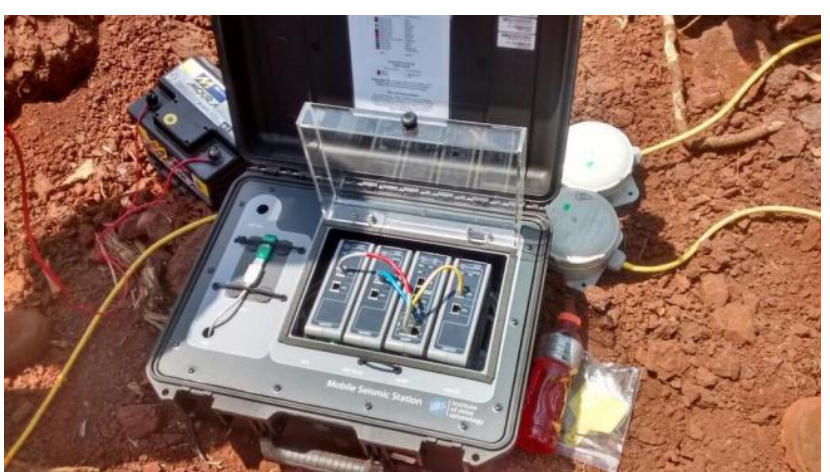

Figure 4 - Mobile seismic station used to record continuous vibrations measured by the geophones.

The continuous vibrations recorded by the seismic station were examined to see if any microseismic events or fractures were recorded by the geophones. Since the blasting took place roughly $500 \mathrm{~m}$ from the cave, it did not expect it to cause any failures close to the cave.

This was indeed the case and no fractures were recorded in the vicinity of the N4E_0026. It is quite possible that fractures do occur on rare occasions in the cave, but that we did not record data for a long enough time to see it. If blasting takes place closer to the cave, it was expected that some fractures would occur in the cave and therefore that conventional microseismic monitoring could provide valuable information. 
Although it did not recorded any fractures in the caves, there were a number of microseismic events recorded by the seismic station that originated far away from the cave. An example of one of these microseismic events is shown in Figure 5.

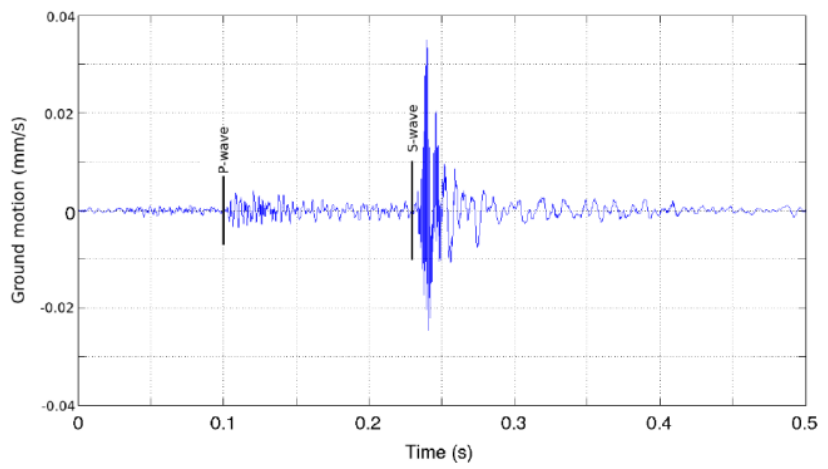

Figure 5-Microseismic event recorded by the seismic monitoring network. The difference in the arrival time between the $P$ - and $S$-waves indicates the event happened far away.

If we consider a reasonable values for the $\mathrm{P}$ - and $\mathrm{S}$-wave velocities of $3600 \mathrm{~m} / \mathrm{s}$ and $2200 \mathrm{~m} / \mathrm{s}$ respectively [2], then the difference in the $\mathrm{P}$ and $\mathrm{S}$-wave arrival times in Figure 5 of 0.17 seconds indicates that this event happened roughly $1 \mathrm{~km}$ away.

Both of the blasts generated seismic signals that were recorded by the sensors. The blast located the closest to the sensors (roughly $500 \mathrm{~m}$ ) generated the strongest ground motions. A seismogram showing the vibrations from the blast are shown in Figure 6. This seismogram was recorded by the sensor closest to the blast (the sensor in the north east in Figure 7).

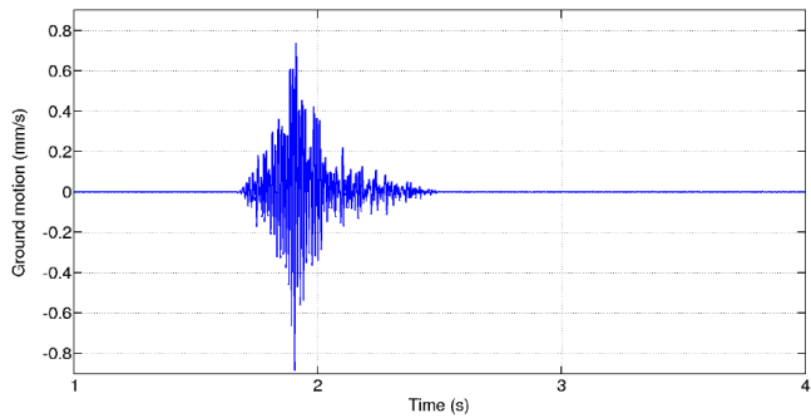

Figure 6 - Vibrations produced by the blast as recorded by one of the seismic sensors. The blast produced peak ground motions of up to $0.9 \mathrm{~mm} / \mathrm{s}$ in this location.

From the seismogram, it was possible to see that the peak ground motion generated by the blast at this sensor was roughly $0.9 \mathrm{~mm} / \mathrm{s}$. The examination of the continuous ground motions indicated that the blast did not generated any detectable aftershocks in the vicinity of the sensors.

The application of the continuous passive monitoring revealed that this method is well suited for monitoring of physical stability of natural caves. The quality of the data was good enough to make hourly (or better) measurement of the seismic velocity in the vicinity of the caves. The velocity changes for each sensor, is shown in Figure 7 for 8 hours before and after the strongest blast.

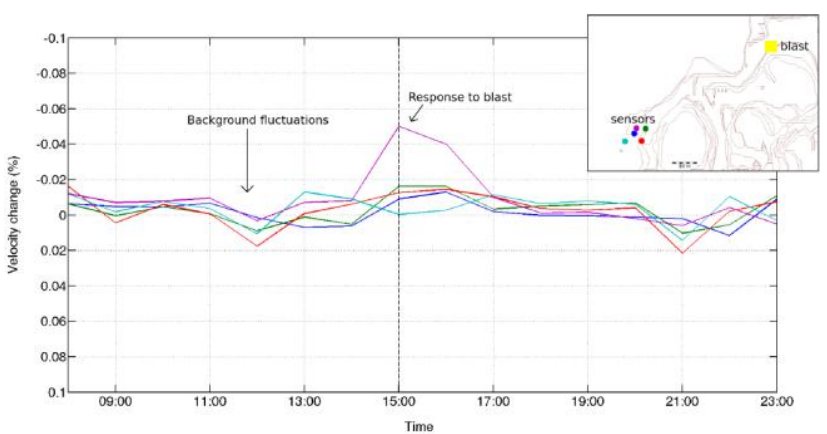

Figure 7-Changes in seismic velocity during the strongest blast. Only one of the sensors (purple) show a response that is larger than the background levels, while the sensors close to the cave see no effects.

From the Figure 7 it is possible to see that the blast caused a small response in the locations of the seismic sensors. The purple sensor experienced the largest change in velocity and the velocity took two hours to return to the level it was before the blast. This effect is quite small compared to the others reported in the literature [3, 4]. The sensors closest to the cave (light blue) did not experience any change in seismic velocity above the background fluctuations. This indicates that the effect of the blast on the cave was smaller than the background effects due to natural phenomena, like temperature change.

Even though the effects of the blast were small, it is possible to image the effects to show how these results would be displayed in periodically monitoring reports. The immediate effects in the area of the cave and sensors are shown in Figure 8 along with the effects two hours after the blast. This shows that the velocities have returned to background levels after 2 hours and that no permanent changes were caused by the blast.
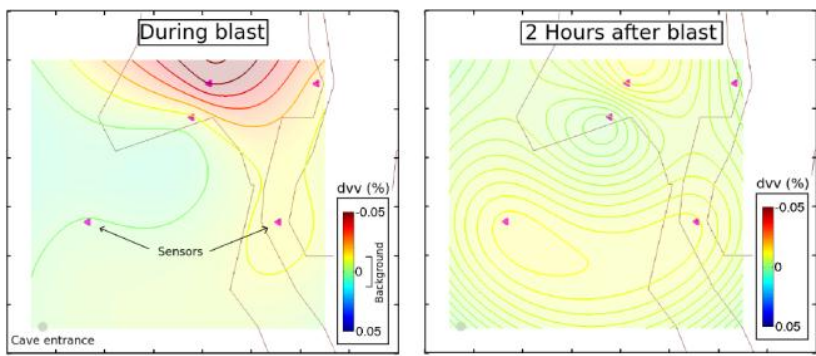

Figure $8-A$ map of the velocity changes (dvvdifference velocity variations) measured immediately after the blast (left) and 2 hours after the blast (right). The sensors closest to the blast see a temporary velocity change during the blast, but after 2 hours this response disappears. The sensors close to the cave see no change. 


\section{Discussion and Conclusions}

These preliminary results showed that microseismic monitoring technology is ideal to continuously and remotely monitor the possible physical impacts that mine operations can cause on damaging the surrounding rock mass that the natural caves within.

This technology allows the identification of unfavorable geological structures present in the caves, enabling to monitor the behavior of these structures over time, with the possibility of differentiating between natural unstable causes, due to weathering processes assignable to the natural evolution of the caves, those due to mining activities.

The seismogram obtained for microseismic activity and blasting (Figure 5 and 6 ) showed a good response to the geological and structural environment surrounding the N4E_0026, generated from the waves produced by the two detonations carried out.

During the process of data acquisition, two blasts were performed. From these detonations, only the closest had an effect on the sensors with higher values than the background noise. This effect was observed in the near of the sensors detonations, no effect was observed in the nearest sensor N4E_0026. The effect disappeared recorded about 2 hours after detonation, indicating that there was no permanent damage in the vicinity of seismic sensors network.

The use of microseismic techniques (that are routinely used in mining operations) to monitor possible physical damage in natural structures like the caves of Carajás due to repeated blasts over a period of time can be a valuable tool in areas where many caves are presented.

The adoption of such technology will allow sustainable relationship between the protection of natural caves and the production of iron ore.

The microseismic monitoring systems and analysis techniques (conventional or passive) will continuously collect information about the rock mass conditions and stability, to inform the mine operators of possible environmental impacts of mining operations.

The implementation of a permanent monitoring network will enable the continuous monitoring of the physical condition of the natural caves along the whole area of the mine operations. In addition, this system will (a) assist in the identification and mapping of new geological structures, (b) definition of possible causes of instabilities, (c) implementation of an early warning system to the physical stability risk of caves collapse and (d) perform the seismic monitoring of the rock mass for large areas, including a range of structures, whether natural or those built by the mining operations like tailings dams and open cut slopes.

The results of the pilot project showed the feasibility of the development of a permanent monitoring network in Carajás. Once the technology has proven to be useful to this type of monitoring, the system can be expanded as required to monitor many caves and the entire mining area.

\section{Acknowledgment}

The authors would like to thank the following professionals for their contribution in the field work for the data acquisition, as follow: Márcio Mansur (Vale) and Luiz Mendonça Amorim (Vale).

\section{References}

[1] Milne, T. and Weir-Jones, A., 2013. Use of passive microseismic monitoring. In: Canadian Mining Journal.

[2] Mendecki, A. J., 1997. Seismic monitoring in mines. Springer Science \& Business Media.

[3] Brenguier, F., Campillo, M., Hadziioannou, C., Shapiro, N., Nadeau, R. and Larose, E., 2008. Postseismic relaxation along the San Andreas fault at Parkfield from continuous seismological observations. Science, 321:1478-1481.

[4] Olivier, G., Brenguier, F., F., Campillo, Roux, P., Shapiro, N. and Lynch, D., 2015. Investigation of co- and post-seismic processes with in-situ measurements of seismic velocity variations in an underground mine. Geophysical Research Letter. 